\title{
A Manifold Learning Approach to Data-Driven Computational Elasticity and Inelasticity
}

\author{
Rubén Ibañez $^{1} \cdot$ Emmanuelle Abisset-Chavanne $^{1} \cdot$ Jose Vicente Aguado $^{1} \cdot$ \\ David Gonzalez $^{2} \cdot$ Elias Cueto $^{2} \cdot$ Francisco Chinesta $^{1}$
}

Received: 4 October 2016/ Accepted: 13 October 2016

(C) CIMNE, Barcelona, Spain 2016

\begin{abstract}
Standard simulation in classical mechanics is based on the use of two very different types of equations. The first one, of axiomatic character, is related to balance laws (momentum, mass, energy,...), whereas the second one consists of models that scientists have extracted from collected, natural or synthetic data. Even if one can be confident on the first type of equations, the second one contains modeling errors. Moreover, this second type of equations remains too particular and often fails in describing new experimental results. The vast majority of existing models lack of generality, and therefore must be constantly adapted or enriched to describe new experimental findings. In this work we propose a new method, able to directly link data to computers in order to perform numerical simulations. These simulations will employ axiomatic, universal laws while minimizing the need of
\end{abstract}

Francisco Chinesta

Francisco.Chinesta@ec-nantes.fr

Rubén Ibañez

Ruben.Ibanez-Pinillo@ec-nantes.fr

Emmanuelle Abisset-Chavanne

Emmanuelle.Abisset-Chavanne@ec-nantes.fr

Jose Vicente Aguado

Jose.Aguado-Lopez@ec-nantes.fr

David Gonzalez

gonzal@unizar.es

Elias Cueto

ecueto@unizar.es

1 High Performance Computing Institute and ESI GROUP Chair, Ecole Centrale de Nantes, 1 Rue de la Noe, 44300 Nantes, France

2 Aragon Institute of Engineering Research, Universidad de Zaragoza, Zaragoza, Spain explicit, often phenomenological, models. This technique is based on the use of manifold learning methodologies, that allow to extract the relevant information from large experimental datasets.

\section{Introduction}

Big Data has bursted in our lives in many aspects, ranging from e-commerce to social sciences, mobile communications, healthcare [16], etc. However, very little has been done in the field of scientific computing, despite some very promising first attempts. Engineering sciences, however, and particularly Integrated Computational Materials Engineering (ICME) [12], seem to be a natural field of application.

In the past, models were more abundant than data, too expensive to be collected and analyzed at that time. However, nowadays, the situation is radically different, data is much more abundant (and accurate) than existing models, and a new paradigm is emerging in engineering sciences and technology. For instance, high-energy physics experiments produce some $1 \mathrm{~Pb}$ of data per day, while in 2012, 162,000 papers were published in materials science and engineering journals.

Advanced clustering techniques, for instance, not only help engineers and analysts, they become crucial in many areas where models, approximation bases, parameters, etc. are adapted depending on the local state (in space and time senses) of the system $[1,9]$. They make possible to define hierarchical and goal-oriented modeling. Machine learning [8] needs frequently to extract the manifold structure in which the solution of complex and coupled engineering problems is living. Thus, uncorrelated parameters can be efficiently extracted from the collected data, coming from 
numerical simulations or experiments. As soon as uncorrelated parameters are identified (constituting the information level), the solution of the problem can be predicted at new locations of the parametric space, by employing adequate interpolation schemes $[5,10]$. On a different setting, parametric solutions can be obtained within an adequate framework able to circumvent the curse of dimensionality for any value of the uncorrelated model parameters [4].

This unprecedented possibility of directly determine knowledge from data or, in other words, to extract models from experiments in a automated way, is being followed with great interest in many fields of science and engineering. For instance, the possibility of fitting the data to a particular set of models has been explore recently in [2]. Willcox and coworkers, on the contrary, have established a strategy that allows to construct reduced-order models from data, by inferring the full-order operators without the need to construct them explicitly, nor having a direct knowledge on the governing models [13, 14]. Closely related, Ortiz has developed a method that works without constitutive models, by finding iteratively the experimental datum that best satisfies conservation laws [6].

In the ICME framework of materials modeling, design, simulation, and manufacturing, this subtle circle is closed by linking data to information, information to knowledge and finally knowledge to real time decision-making, opening unprecedented possibilities within the so-called DDDAS (Dynamic Data Driven Application Systems) $[3,11]$.

In the present work we will assume that all the needed data is available. We will not address all the difficulties related to data generation or obtention from adequate experiments. This is a topic that, of course, remains open. On the contrary, we develop a method in which this stream of data plays the role of a constitutive equation, without the need of a phenomenological fitting to a prescribed model.

To better understand the data-driven rationale addressed in the present paper, let us consider, for the sake of clarity, a very simple problem: linear elasticity. In that case the balance of (linear and angular) momentum leads to the existence of a symmetric second-order tensor $\sigma$ (the socalled Cauchy's stress tensor) verifying equilibrium, expressed in the absence of body forces, as

$\nabla \cdot \boldsymbol{\sigma}=\boldsymbol{0}$.

The finite-element solution of this equilibrium equation starts from establishing a weak form in the domain $\Omega$ with boundary $\Gamma \equiv \partial \Omega$,

$\int_{\Omega} \boldsymbol{u}^{*} \cdot(\nabla \cdot \boldsymbol{\sigma}) d \boldsymbol{x}=0$.

By integrating by parts, it results
$\int_{\Omega} \nabla \boldsymbol{u}^{*}: \boldsymbol{\sigma} d \boldsymbol{x}=\int_{\Gamma} \boldsymbol{u}^{*} \cdot(\boldsymbol{\sigma} \cdot \boldsymbol{n}) d \boldsymbol{x}$,

where $\boldsymbol{n}$ represents the outward unit vector normal to the boundary.

If we consider $\Gamma=\Gamma_{D} \cup \Gamma_{N},\left(\Gamma_{D} \cap \Gamma_{N}=\emptyset\right)$, representing portions of the domain boundary where, respectively, displacements $\boldsymbol{u}=\boldsymbol{u}_{g}(\boldsymbol{x})$ (Dirichlet boundary conditions) and tractions $\boldsymbol{\sigma} \cdot \boldsymbol{n}=\boldsymbol{t}_{g}(\boldsymbol{x})$ (Neumann boundary conditions) are enforced, the weak form finally reads:

Find the displacement field $\boldsymbol{u} \in\left(\mathcal{H}^{1}(\Omega)\right)^{3}$ satisfying the essential boundary conditions $\boldsymbol{u}\left(\boldsymbol{x} \in \Gamma_{D}\right)=\boldsymbol{u}_{g}(\boldsymbol{x})$ such that

$\int_{\Omega} \boldsymbol{\varepsilon}^{*}: \boldsymbol{\sigma} d \boldsymbol{x}=\int_{\Gamma_{N}} \boldsymbol{u}^{*} \cdot \boldsymbol{t} d \boldsymbol{x}$,

$\forall \boldsymbol{u}^{*}$ regular enough and vanishing on $\Gamma_{D}$, i.e. $\forall \boldsymbol{u}^{*} \in\left(\mathcal{H}_{0}^{1}(\Omega)\right)^{3}$.

In the previous weak form, the symmetry of $\sigma$ implies the equality $\nabla \boldsymbol{u}: \boldsymbol{\sigma}=\nabla^{S} \boldsymbol{u}: \boldsymbol{\sigma}$, with $\nabla^{S} \boldsymbol{u}$ the symmetric component of the displacement gradient, also known as strain tensor, generally denoted by $\boldsymbol{\varepsilon}$.

The weak form given by Eq. (1) involves kinematic and dynamic variables from the test displacement field $\boldsymbol{u}^{*}$ and the stress tensor $\boldsymbol{\sigma}$ respectively. In order to solve it a relationship linking kinematic and dynamic variables is required, the socalled constitutive equation. The simplest one, giving rise to linear elasticity, is known as Hooke's law (even if, more than a law, it is simply a model), and writes

$\boldsymbol{\sigma}=\lambda \operatorname{Tr}(\boldsymbol{\varepsilon}) \boldsymbol{I}+\mu \boldsymbol{\varepsilon}$

where $\operatorname{Tr}(\cdot)$ denotes the trace operator, and $\lambda$ and $\mu$ are the Lame coefficients directly related to the Young modulus $E$ and the Poisson coefficient $v$.

By introducing the constitutive model, Eq. (2), into the weak form of the balance of momentum, Eq. (1), a problem is obtained that can be formulated entirely in terms of the displacement field $\boldsymbol{u}$. By discretizing it, using standard finite element approximations, for instance, and performing numerically the integrals involved in Eq. (1), we finally obtain a linear algebraic system of equations, from which the nodal displacements can be obtained.

In the case of linear elasticity there is no room for discussion: the approach is simple, efficient and has been applied successfully to many problems of interest. Today, there are numerous commercial codes making use of this mechanical behavior and nobody doubts about its pertinence in engineering practice. However, there are other material behaviors for whom simple models fail to describe any experimental finding. These models lack of generality (universality) and for this reason a mechanical system is usually associated to different models that are progressively adapted and/or enriched from the collected data. 
The biggest challenge could then be formulated as follows: can simulation proceed directly from data by circumventing the necessity of establishing a constitutive model? In the case of linear elasticity it is obvious that such an approach lacks of interest. However, in other branches of engineering science and technology it should be an appealing alternative to standard constitutive model-based simulations. In our opinion, we are at the beginning of a new era, the one of data-based or, more properly, datadriven engineering science and technology, where as much as possible data should be collected and information extracted in a systematic way by using adequate machine learning strategies. Then, simulations could proceed directly from this automatically acquired knowledge.

Thus, the question from a methodological viewpoint could be reformulated as: If Hooke had never existed, linear elasticity finite element simulations would have existed?

This paper addresses this question, trying to push it beyond linear elastic behaviors. Next section focuses on the construction of the so-called constitutive manifold from the collected data. Section 3 defined the manifold-based datadriven framework, and Sect. 4 introduces data-driven simulation in the context of elastic models (linear and nonlinear). Finally, Sect. 5 extends the procedure to inelastic behaviors.

\section{Collecting Data and Constructing the Constitutive Manifold}

Imagine, to begin with (more general scenarios will soon be considered) mechanical tests conducted on a perfectly linear elastic material, in a specimen exhibiting uniform stresses and strains. As previously indicated, in this paper we do not address issues related to data generation. Thus, for $M$ randomly applied external loads, we assume ourselves able to collect $M$ couples $\left(\boldsymbol{\sigma}_{m}, \boldsymbol{\varepsilon}_{m}\right), m=1, \ldots, M$. These pairs could be represented as a single point $\boldsymbol{X}_{m}$ in a phase space of dimension $D=12$ (the six distinct components of the stress and strain tensors, respectively). In the sequel Voigt notion will be considered, i.e. stress and strain tensors will be represented as vectors and the fourth-order elastic tensor reduces to a square matrix.

Each vector $\boldsymbol{X}_{m}$ thus defines a point in a space of dimension $D$ and, therefore, the whole set of samples represents a set of $M$ points in $\mathbb{R}^{D}$. We conjecture that all these points belong to a certain low-dimensional manifold embedded in the high-dimensional space $\mathbb{R}^{D}$. Imagine for a while that the $M$ points belong to a curve, a surface or a hyper-surface of dimension $d \ll D$. When $D=3$ a simple observation suffices for checking if these points are located on a curve (one-dimensional manifold) or on a surface (two-dimensional manifold). However, when dealing with high dimensional spaces, a simple visual observation is, in general, not possible. Moreover, the extraction of uncorrelated features (often referred to as latent parameters) seems to be more physically pertinent.

Therefore, appropriate manifold learning (or non-linear dimensionality reduction) techniques are needed to extract the underlying manifold (when it exists) in multidimensional phase spaces. A panoply of techniques exist to this end. The interested reader can refer to [1, 15, 17-19], just to cite a few references. In this work we focus on the particular choice of Locally Linear Embedding--LLEtechniques [17]. This method proceeds in two steps:

1. Each point $\boldsymbol{X}_{m}, m=1, \ldots, M$ is linearly interpolated from its $K$ nearest neighbors. In principle $K$ should be greater that the expected dimension $d$ of the underlying manifold and the neighbors should be close enough so as to ensure the validity of linear approximation. In general, a small but enough number of neighbors $K$ and a large-enough sampling $M$ ensures a satisfactory reconstruction. For each point $\boldsymbol{X}_{m}$ we can write the locally linear data reconstruction as:

$\boldsymbol{X}_{m}=\sum_{i \in \mathcal{S}_{m}} W_{m i} \boldsymbol{X}_{i}$

where $W_{m i}$ are the unknown weights and $\mathcal{S}_{m}$ the set of the $K$-nearest neighbors of $\boldsymbol{X}_{m}$.

If we perform this locally linear interpolation for every data point in the high dimensional phase space, the set of weights that best approximates the manifold structure of the data will be obtained by minimizing the functional

$\mathcal{F}(\boldsymbol{W})=\sum_{m=1}^{M}\left\|\boldsymbol{X}_{m}-\sum_{i=1}^{M} W_{m i} \boldsymbol{X}_{i}\right\|^{2}$,

where $W_{m i}$ is zero if $\boldsymbol{X}_{i}$ does not belong to the set of $K$ nearest neighbors of $\boldsymbol{X}_{m}$.

2. We assume now that each linear patch around $\boldsymbol{X}_{m}, \forall m$, is mapped onto a lower dimensional embedding space of dimension $d \ll D$. To maintain the neighborhood structure of the set (other methods like isomap [19] conserve distance in the embedding space instead), weights are assumed to remain unchanged in the lowdimensional, embedding space. The problem thus becomes the determination of the coordinates of each point $\boldsymbol{X}_{m}$ in the low dimensional embedding space, $\boldsymbol{\xi}_{m} \in \mathbb{R}^{d}$.

For this purpose a new functional $\mathcal{G}$ is introduced, that depends on the searched coordinates $\boldsymbol{\xi}_{1}, \ldots, \boldsymbol{\xi}_{M}$ 
$\mathcal{G}\left(\boldsymbol{\xi}_{1}, \ldots, \boldsymbol{\xi}_{M}\right)=\sum_{m=1}^{M}\left\|\boldsymbol{\xi}_{m}-\sum_{i=1}^{M} W_{m i} \xi_{i}\right\|^{2}$

where now the weights are known and the reduced coordinates $\xi_{m}$ are unknown. The minimization of functional $\mathcal{G}$ results in a $M \times M$ eigenvalue problem whose $d$-bottom non-zero eigenvalues define the set of orthogonal coordinates in which the manifold is mapped.

It is important to note that functional $\mathcal{G}\left(\boldsymbol{\xi}_{1}, \ldots, \boldsymbol{\xi}_{M}\right)$, with the different coordinates $\boldsymbol{\xi}_{m}$ already calculated as just described, offers an error estimator on the locally linear embedding capacity, and even a local estimator can be derived by considering

$$
\mathcal{E}\left(\xi_{m}\right)=\left\|\xi_{m}-\sum_{i=1}^{M} W_{m i} \xi_{i}\right\| .
$$

Thus, if we consider the introduction of a new point $\xi$ in the embedding space $\mathbb{R}^{d}$ after identifying its neighbors set $\mathcal{S}(\xi)$ and calculating the locally linear approximation weights, we can come back to $\mathbb{R}^{D}$ and reconstruct $\boldsymbol{X}$ from its neighbors $\boldsymbol{X}_{i}, i \in \mathcal{S}(\xi)$.

In the linear elastic behavior the application of the just described technique results, as expected, in a flat manifold of dimension two, i.e. $d=2$. This is in perfect agreement to the fact that Hooke's law is completely characterized by two coefficients (either Young's modulus and Poisson coefficient, or Lame's coefficients) and is linear. Figure 1

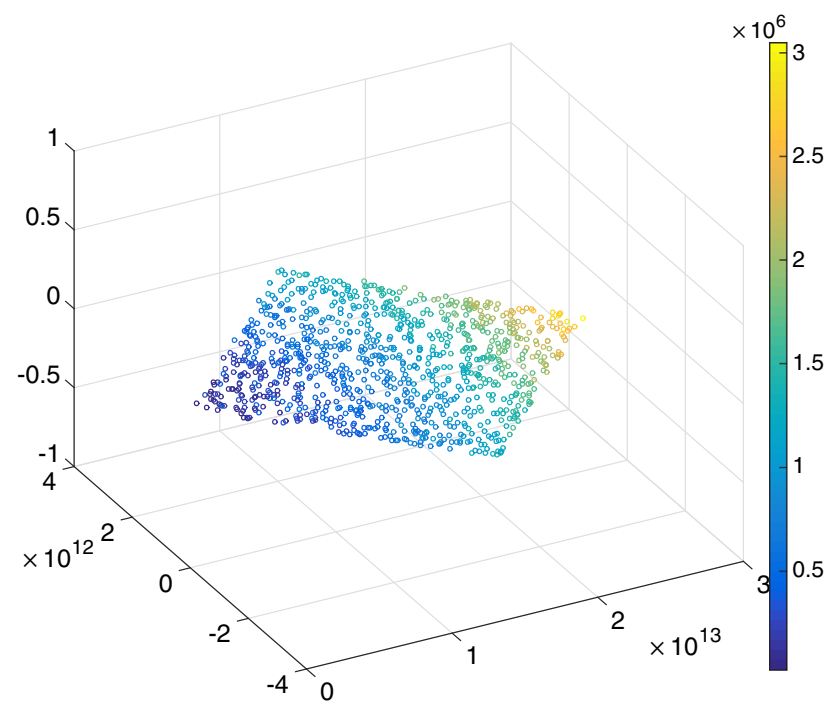

Fig. 1 Reduced coordinates $\xi_{m}$ on the resulting two-dimensional manifold. The color map represents the associated elastic energy. (Color figure online) depicts the location of samples $\xi_{m}=\boldsymbol{\xi}\left(\mathbf{X}_{\mathbf{m}}\right)=\boldsymbol{\xi}\left(\boldsymbol{\sigma}_{\mathbf{m}}, \boldsymbol{\varepsilon}_{\mathbf{m}}\right)$ into the resulting two-dimensional manifold, as well as the associated elastic energy of each sample, showing that LLE preserves the smoothness of the elastic energy field of the sample in the embedding space.

\section{Working with Constitutive Manifolds}

We have abandoned the idea of a phenomenological constitutive equation. Instead, we have defined the concept of (experimentally obtained) constitutive manifold, as the one with a minimal number of latent parameters (embedding coordinates) in which the state of the sample will evolve in different stress and strain conditions.

However, for the method to be useful, we need to define a strategy to solve problems stated in weak form and discretized by finite elements. Several options can be considered, which are described next.

1. Identifying the locally linear behavior. If we consider locally linear approximations, fully justified if $\mathcal{E}\left(\boldsymbol{\xi}_{m}\right)$, given by Eq. (4), remains small enough at each position $\xi_{m}$ (if it is not the case the sampling should be improved locally or globally), we can write

$\xi_{m}=\sum_{i=1}^{M} W_{m i} \xi_{i}$

with $W_{m i}=0$ if $i \notin \mathcal{S}_{m}$ and where $\xi_{m}$ is a stress-strain couple. This implies a locally linear elastic behavior, that allows obtaining the elastic tensor $\boldsymbol{C}$ from $\boldsymbol{X}_{m}$ and $\boldsymbol{X}_{i}$ (related to $\boldsymbol{\xi}_{m}$ and $\boldsymbol{\xi}_{i}$ respectively), with $i \in \mathcal{S}_{m}$, by minimizing the functional

$$
\mathcal{H}(\boldsymbol{C})=\sum_{i \in \mathcal{S}_{m}}\left(\boldsymbol{\sigma}_{i}-\boldsymbol{C} \cdot \boldsymbol{\varepsilon}_{i}\right)^{2}
$$

This results in the obtention of $\boldsymbol{C}\left(\boldsymbol{X}_{m}\right) \equiv \boldsymbol{C}_{m}$.

2. Identifying the locally linear tangent behavior. In order to consider Newton strategies the locally tangent linear behavior should be computed. Again, it is easy to obtain by considering $\Delta_{m i} \equiv \boldsymbol{X}_{m}-\boldsymbol{X}_{i}=\left(\boldsymbol{\sigma}_{m}-\right.$ $\left.\boldsymbol{\sigma}_{i}, \boldsymbol{\varepsilon}_{m}-\boldsymbol{\varepsilon}_{i}\right)$ or $\Delta_{m i}=\left(\Delta \boldsymbol{\sigma}_{i}^{m}, \Delta \boldsymbol{\varepsilon}_{i}^{m}\right), i \in \mathcal{S}_{m}$. Because of the locally linear behavior around point $\boldsymbol{X}_{m}$, we can write

$\Delta \boldsymbol{\sigma}_{i}^{m}=\boldsymbol{C}_{T} \cdot \Delta \varepsilon_{i}^{m}$,

that allows defining the functional $\mathcal{H}_{T}\left(\boldsymbol{C}_{T}\right)$

$\mathcal{H}_{T}\left(\boldsymbol{C}_{T}\right)=\sum_{i \in \mathcal{S}_{m}}\left(\Delta \boldsymbol{\sigma}_{i}^{m}-\boldsymbol{C}_{T} \cdot \Delta \boldsymbol{\varepsilon}_{i}^{m}\right)^{2}$,

whose minimization results in the tangent elastic tensor $\boldsymbol{C}_{T}\left(\boldsymbol{X}_{m}\right) \equiv \boldsymbol{C}_{T, m}$. 
3. No model at all. The third level of description considers points $\boldsymbol{X}_{m}$ without trying to identify local behavior models at all.

It is important to note that even if the just discussed descriptions are based on the original manifold $\boldsymbol{X}_{m}$ and not on the reduced one $\boldsymbol{\xi}_{m}$, the consideration of the reduced manifold allows to obtain a global view of the manifold dimensionality as well as safer interpolations on the manifold. This ensures that interpolated data $\xi$ belongs to the manifold, before applying the inverse mapping to obtain $\boldsymbol{X}$ on the original manifold.

\section{Data-Driven Simulation in the Elastic Case}

We assume that the elastic behavior is accessible from the data contained into the so-called constitutive manifold but that an explicit expression relating stresses and strains is neither available nor desired. Immediately, a question arises on how to solve the weak form related to the equilibrium of the mechanical system given by Eq. (1) if no closed-form expression on $\boldsymbol{\sigma}=\boldsymbol{\sigma}(\boldsymbol{\varepsilon})$ is available.

\subsection{Discretization Schemes}

In this case we could consider three different approaches depending on the chosen behavior description as just discussed in the previous section:

1. From the just identified locally linear behavior $\boldsymbol{C}(\boldsymbol{X})$ one could apply the simplest explicit linearization technique operating on the standard weak form

$\int_{\Omega} \boldsymbol{\varepsilon}^{*}(\boldsymbol{x}): \boldsymbol{\sigma}^{n+1}(\boldsymbol{x}) d \boldsymbol{x}=\int_{\Gamma_{N}} \boldsymbol{u}^{*}(\boldsymbol{x}) \cdot \boldsymbol{t}(\boldsymbol{x}) d \boldsymbol{x}$,

where at each point, from the stress-strain couple at position $\boldsymbol{x}, \boldsymbol{X}(\boldsymbol{x})$, the locally linear behavior $\boldsymbol{C}(\boldsymbol{X}(\boldsymbol{x}))$ can be obtained (in practice at the Gauss points used for the integration of the weak form) that allows us to write (using Voigt notation)

$\int_{\Omega} \boldsymbol{\varepsilon}^{*}(\boldsymbol{x}) \cdot(\boldsymbol{C}(\boldsymbol{x}) \cdot \boldsymbol{\varepsilon}(\boldsymbol{x})) d \boldsymbol{x}=\int_{\Gamma_{N}} \boldsymbol{u}^{*}(\boldsymbol{x}) \cdot \boldsymbol{t}(\boldsymbol{x}) d \boldsymbol{x}$.

This allows, in turn, to compute the displacement field and from it, to update the strain and stress fields, to compute again the locally linear behavior. The process continues until convergence.

2. From the just identified locally linear tangent behavior $\boldsymbol{C}_{T}(\boldsymbol{X})$ one could apply a Newton linearization technique where

$\boldsymbol{\sigma}(\boldsymbol{\varepsilon}+\Delta \varepsilon)=\boldsymbol{\sigma}(\boldsymbol{\varepsilon})+\frac{\partial \boldsymbol{\sigma}}{\partial \boldsymbol{\varepsilon}} \Delta \varepsilon=\boldsymbol{\sigma}(\boldsymbol{\varepsilon})+\boldsymbol{C}_{T} \cdot \Delta \varepsilon$, that, once introduced into the weak form, reads

$$
\begin{aligned}
& \int_{\Omega} \boldsymbol{\varepsilon}^{*}(\boldsymbol{x}) \cdot\left(C_{T}(\boldsymbol{x}) \cdot \Delta \boldsymbol{\varepsilon}(\boldsymbol{x})\right) d \boldsymbol{x} \\
& \quad=-\int_{\Omega} \boldsymbol{\varepsilon}^{*}(\boldsymbol{x}) \cdot(\boldsymbol{C}(\boldsymbol{x}) \cdot \boldsymbol{\varepsilon}(\boldsymbol{x})) d \boldsymbol{x}+\int_{\Gamma_{N}} \boldsymbol{u}^{*}(\boldsymbol{x}) \cdot \boldsymbol{t}(\boldsymbol{x}) d \boldsymbol{x} .
\end{aligned}
$$

3. If no local behavior has been identified, the only knowledge consists of the experimental data. In these circumstances we propose to consider a mixed formulation involving the two unknown fields $\boldsymbol{\varepsilon}(\boldsymbol{u})$ and $\boldsymbol{\sigma}$ as considered in the LaTIn method [8]. We consider a simple solution strategy consisting on an iteration between two manifolds, the first one related to $(\boldsymbol{\varepsilon}, \boldsymbol{\sigma})$ couples verifying equilibrium Eq. (1); and the second one related to couples $(\hat{\boldsymbol{\varepsilon}}, \hat{\boldsymbol{\sigma}})$ verifying the (unknown) constitutive equation-in other words, belonging to the constitutive manifold. The iteration solver sketched in Fig. 2, depicts the usually non linear constitutive manifold (red curve) and the equilibrium one (blue straight line). The problem solution is found at the intersection of both manifolds.

If we assume that, at iteration $n$, the couple $\left(\varepsilon^{n}, \boldsymbol{\sigma}^{n}\right)$ verifies the equilibrium, and that it does not belong to the constitutive manifold, a new couple $(\hat{\boldsymbol{\varepsilon}}, \hat{\boldsymbol{\sigma}})$ is sought by considering an appropriate search direction from $\left(\boldsymbol{\varepsilon}^{n}, \boldsymbol{\sigma}^{n}\right)$. In fact the searched couple is no more that the intersection of the search direction with the constitutive manifold. The just updated stress-strain couple belongs to the constitutive manifold, but it does not verify equilibrium. Thus, a new equilibrated solution $\left(\boldsymbol{\varepsilon}^{n+1}, \boldsymbol{\sigma}^{n+1}\right)$ is searched from the former one, being the intersection of a new search direction and the equilibrium manifold. The iteration process continues until reaching the problem solution at the intersection of both manifolds.

The just described procedure requires a local step for the computation of the couple $(\hat{\boldsymbol{\varepsilon}}, \hat{\boldsymbol{\sigma}})$ at each integration point considered in the weak form, Eq.(1), and a global

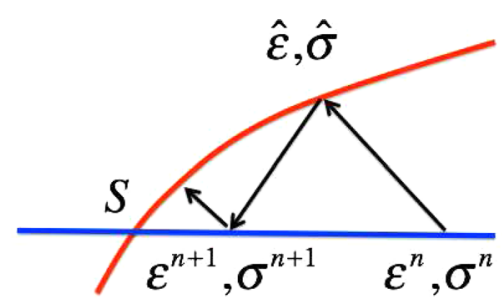

Fig. 2 A generic nonlinear iteration solver between the constitutive manifold (red curve) and the equilibrium manifold (blue straight line), representing the locus of the points satisfying the weak form of the problem in mixed form, Eq. (7). (Color figure online) 
step in which the weak form is solved with the behavior known at all the integration points. In what follows we describe both steps.

- Local step

At each integration point $\boldsymbol{x}_{g}, g=1, \ldots, \mathrm{ngp}$, we consider $\left(\boldsymbol{\varepsilon}^{n}\left(\boldsymbol{x}_{g}\right), \boldsymbol{\sigma}^{n}\left(\boldsymbol{x}_{g}\right)\right)$ and look for $\left(\hat{\boldsymbol{\varepsilon}}\left(\boldsymbol{x}_{g}\right), \hat{\boldsymbol{\sigma}}\left(\boldsymbol{x}_{g}\right)\right)$. Even if there is an infinity of possible search directions, a natural choice consists in projecting it onto the constitutive manifold.

- Global step

From the strain-stress couples satisfying the constitutive law at every integration point, we come back to the weak form, Eq. (1), in order to obtain updated strain-stress couples satisfying equilibrium $\left(\boldsymbol{\varepsilon}^{n+1}(\boldsymbol{x}), \boldsymbol{\sigma}^{n+1}(\boldsymbol{x})\right), \boldsymbol{x} \in \Omega$.

The generic search direction can be written as:

$\boldsymbol{\sigma}^{n+1}(\boldsymbol{x})-\hat{\boldsymbol{\sigma}}(\boldsymbol{x})=\boldsymbol{D} \cdot\left(\boldsymbol{\varepsilon}^{n+1}(\boldsymbol{x})-\hat{\boldsymbol{\varepsilon}}(\boldsymbol{x})\right)$,

with $\boldsymbol{D}$ a symmetric positive-definite matrix to ensure the problem ellipticity discussed below. Enforcing now the equilibrium

$\int_{\Omega} \boldsymbol{\varepsilon}^{*}(\boldsymbol{x}) \cdot \boldsymbol{\sigma}^{n+1}(\boldsymbol{x}) d \boldsymbol{x}=\int_{\Gamma_{N}} \boldsymbol{u}^{*}(\boldsymbol{x}) \cdot \boldsymbol{t}(\boldsymbol{x}) d \boldsymbol{x}$,

and using Eq. (8), it results

$$
\begin{aligned}
\int_{\Omega} \boldsymbol{\varepsilon}^{*}(\boldsymbol{x}) \cdot\left(\hat{\boldsymbol{\sigma}}(\boldsymbol{x})+\boldsymbol{D} \cdot\left(\boldsymbol{\varepsilon}^{n+1}(\boldsymbol{x})-\hat{\boldsymbol{\varepsilon}}(\boldsymbol{x})\right)\right) d \boldsymbol{x} \\
=\int_{\Gamma_{N}} \boldsymbol{u}^{*}(\boldsymbol{x}) \cdot \boldsymbol{t}(\boldsymbol{x}) d \boldsymbol{x}
\end{aligned}
$$

that can be rewritten as

$$
\begin{aligned}
& \int_{\Omega} \boldsymbol{\varepsilon}^{*}(\boldsymbol{x}) \cdot\left(\boldsymbol{D} \cdot \boldsymbol{\varepsilon}^{n+1}(\boldsymbol{x})\right) d \boldsymbol{x}=-\int_{\Omega} \boldsymbol{\varepsilon}^{*}(\boldsymbol{x}) \\
& \cdot(\hat{\boldsymbol{\sigma}}(\boldsymbol{x})-\boldsymbol{D} \cdot \hat{\boldsymbol{\varepsilon}}(\boldsymbol{x})) d \boldsymbol{x}+\int_{\Gamma_{N}} \boldsymbol{u}^{*}(\boldsymbol{x}) \cdot \boldsymbol{t}(\boldsymbol{x}) d \boldsymbol{x} .
\end{aligned}
$$

Matrix $\boldsymbol{D}$ should provide the fastest convergence rate while ensuring the problem ellipticity. To ensure its positivity we can consider $\boldsymbol{D}=\boldsymbol{B}^{2}$ with $\boldsymbol{B}$ symmetric, i.e. $\boldsymbol{B}^{T}=\boldsymbol{B}$, and look for $\boldsymbol{B}$ instead of D.

The a priori choice of direction $\boldsymbol{D}$ is not obvious in most of problems. In the case of the LaTIn method [8] this matrix is assumed given when solving the global problems precisely because it was proposed as a nonlinear solver able to decouple the local and nonlinear problem from the global but linear one. In our case, we are considering a mixed formulation for solving a problem without an explicit knowledge of the constitutive equation. The most general option consists of considering matrix $\boldsymbol{D}$ unknown. Thus, our strategy is composed of a sequence of nonlinear-local and nonlinear-global problems, trying to avoid a priori choices of $\boldsymbol{D}$. Obviously if the last is fixed, global problems become linear as it is the case when considering the LaTIn linearization technique. Moreover, the discrete global matrix does not change during the iterations. However, we would like to emphasize that our objective is to solve a constitutive modelfree problem, more than addressing nonlinear issues.

Thus, we distinguish two type of iterations, the socalled global-local ones that involves the determination of stress-strain couples verifying the constitutive equation and then their updating to ensure equilibrium (as illustrated in Fig. 2). Then a second iteration is needed for solving the nonlinear global problem in order to compute the stress-strain couple verifying equilibrium when the searching direction $\boldsymbol{D}$ is assumed unknown. This induces an additional nonlinearity in the global equilibrium problem.

At this point two possibilities exist:

a. Considering a single direction $\boldsymbol{D}$, the same for every Gauss point for which the behavior was determined. Each of them is represented by a point on the constitutive manifold. In that case in order to determine the stress-strain couple satisfying equilibrium as well as the optimal direction $\boldsymbol{D}$, we are enforcing Eq. (9) as well as the fact that the searched couple $\left(\boldsymbol{\varepsilon}^{n+1}(\boldsymbol{x}), \boldsymbol{\sigma}^{n+1}(\boldsymbol{x})\right)$ must be the closest point to the constitutive manifold. This optimality condition writes

$$
\begin{aligned}
\boldsymbol{D} & =\underset{\boldsymbol{D}^{*}}{\arg \min }\left(\left(\boldsymbol{\sigma}^{n+1}\left(\boldsymbol{x} ; \boldsymbol{D}^{*}\right)-\hat{\boldsymbol{\sigma}}^{*}\right)^{2}\right. \\
& \left.+\left(\boldsymbol{\varepsilon}^{n+1}\left(\boldsymbol{x} ; \boldsymbol{D}^{*}\right)-\hat{\boldsymbol{\varepsilon}}^{*}\right)^{2}\right)
\end{aligned}
$$

where $\left(\hat{\boldsymbol{\sigma}}^{*}, \hat{\boldsymbol{\varepsilon}}^{*}\right)$ is the closest point on the constitutive manifold to the stress-strain couple related to the direction $\boldsymbol{D}^{*}$.

Obviously the solution requires some iterations to reach the minimum distance that will be in general (except when considering linear behaviors) non-zero because we consider the same matrix $\boldsymbol{D}$ for all the Gauss points involved in the integration of the weak form (9).

b. We consider a field $\boldsymbol{D}(\boldsymbol{x})$, that implies the increase of the number of degrees of freedom. However, by considering for example a different matrix at each Gauss point, the minimization problem given by Eq. (10) leads to the 
problem solution in a single iteration. The employ of a coarse mesh to approximate $\boldsymbol{D}$ is a nice compromise between the two limit cases: considering a single search direction or one at each Gauss point.

\subsection{A First Numerical Example: A Beam Subjected to Simple Traction}

In order to illustrate the data-driven procedure, we consider first a linear elastic beam subjected to simple traction and solve the associated 1D equilibrium problem. Different scenarios are considered and discussed below.

First, the beam is assumed clamped at its left boundary $x=0$ with a constant unit force $F=1$ applied at its right boundary $x=1$. Because of the expected simple solution only 5 linear finite elements were considered for discretizing its equilibrium weak form. Figure 3 depicts the
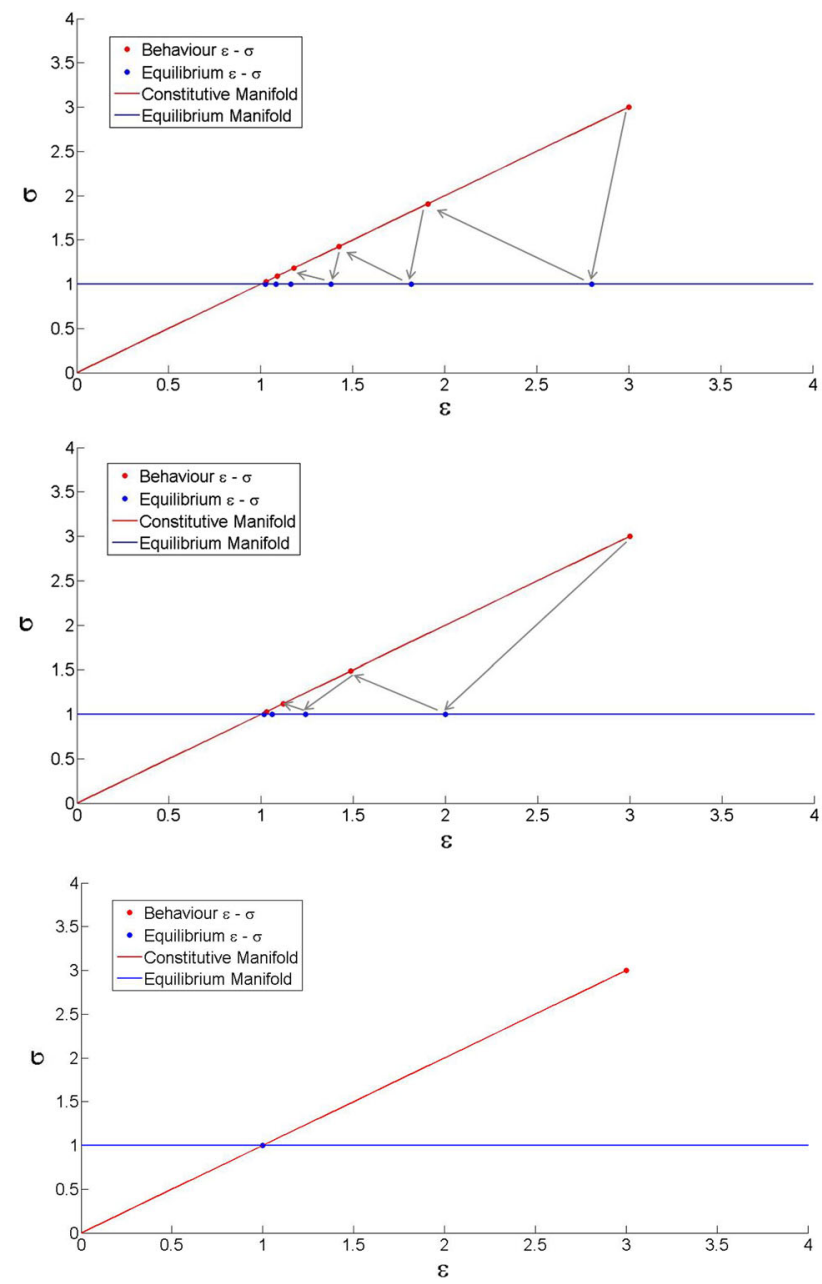

Fig. 3 Beam subjected to traction: (top) $D=10$, (center) $D=2$ and (bottom) $D=1$ constitutive manifold. In a general setting, this manifold should come from experiments, but in this case was generated in silico by assuming a linear elastic behavior with an unit elastic modulus.

The use of strategies based on the identification of the locally linear behavior or its tangent counterpart allows as expected (due to its linear behavior) solving the problem in a single iteration. It is important to note that both strategies are weakly intrusive, making possible its implementation into any commercial simulation code with the only difference that the updated locally linear behavior comes form a data table instead of any mathematical expression.

In what follows we are discussing the use of the third strategy. The equilibrium manifold and the different strainstress couples at the different iterations are depicted in Fig. 3 for $D=10, D=2$ and $D=1$. These $D$-values represent in fact different search directions in Fig. 2. It can be noticed that when $D=1$ is chosen, this value coincides with the elastic modulus associated to the constitutive manifold, and therefore convergence is reached in a single iteration. All the simulations started by assuming the same stress-strain couple $\left(\sigma^{0}, \varepsilon^{0}\right)=(3.0,3.0)$ at every Gauss point.

In these figures, the search direction in the global problem $D$ was fixed "a priori". When the strategy described in the previous section is used, implying the determination of the optimal value of $D$, the nonlinear problem involving $\sigma, \quad \varepsilon$ and $D$, with $\left(\sigma^{0}=3, \varepsilon^{0}=3, D^{0}=3\right)$, converges in a single iteration of the local-global problem. This is so even if a few iterations were required for solving the nonlinear global problem, to obtain the reference values defining the problem solution ( $\sigma=1.0, \varepsilon=1.0, D=1)$. Because of the linearity of the constitutive manifold, no difference exists between considering a single direction $D$ or a different one at each Gauss point. The solution is again obtained in a single global-local iteration and a few ones for solving the nonlinear global problem.

In order to make the problem a bit more complex, we consider the previous one but now we consider an uniformly distributed traction along the beam length. Thus a linear stress and strain distribution is expected. In other words, each Gauss point will be at a state located at different points of the constitutive manifold. Figure 4 represents the stress-strain manifold along the beam length, where the stress-strain couples at the Gauss points are shown. It can be seen that when starting from the initial guess $\left(\sigma^{0}(x)=3, \varepsilon^{0}(x)=3, D^{0}=3\right)$ and again because of the linearity of the constitutive manifold, the convergence is reached in a single global-local iteration with few iterations for the solution of the nonlinear global problem. 


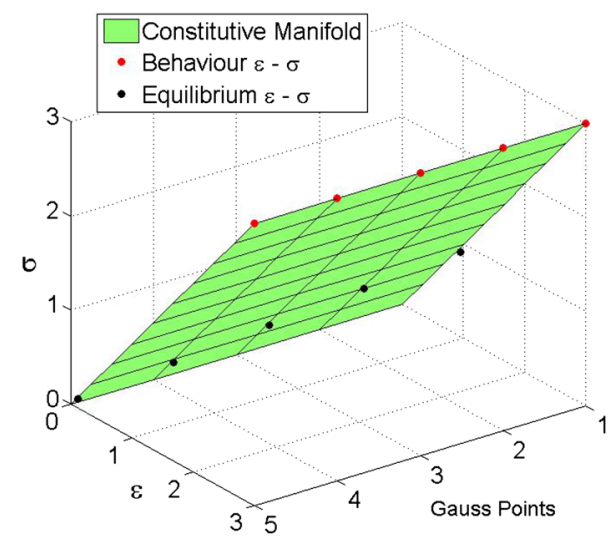

Fig. 4 Beam subjected to uniformly distributed traction

Finally, we consider a nonlinear constitutive law defined from points with a prescribed stress-strain relationship $\sigma=E \epsilon^{2}$, with $E=1$. In the case of a unit traction at the right boundary and when considering uniform initial strain and stress guesses on the constitutive manifold, all the Gauss points will have an identical behavior.

When applying the fixed point linearization based on the locally linear manifold $\boldsymbol{C}$ or the Newton strategy making use of the locally linear tangent manifold $\boldsymbol{C}_{T}$, the procedure proposed in the previous section converges very fast. Iterations to convergence are depicted in Figs. 5 and 6 respectively.

If, on the contrary, we proceed following the third strategy mentioned previously, i.e., directly from data, Fig. 7 depicts the initial guess and the solution after convergence $(\sigma(x)=1, \epsilon(x)=1)$. Here, $D$ is unique and calculated at each global-local iteration. Moreover, at each one of these iterations a nonlinear global problem must be solved needing for few extra-iterations.

If we combine behavior nonlinearities and nonuniform solutions (e.g., a distributed traction along the bar) we proved that the convergence can be improved by considering a different $D$ at each Gauss point with respect to the use of a single search direction $D$ for all them, even if the global problem size increases significantly.

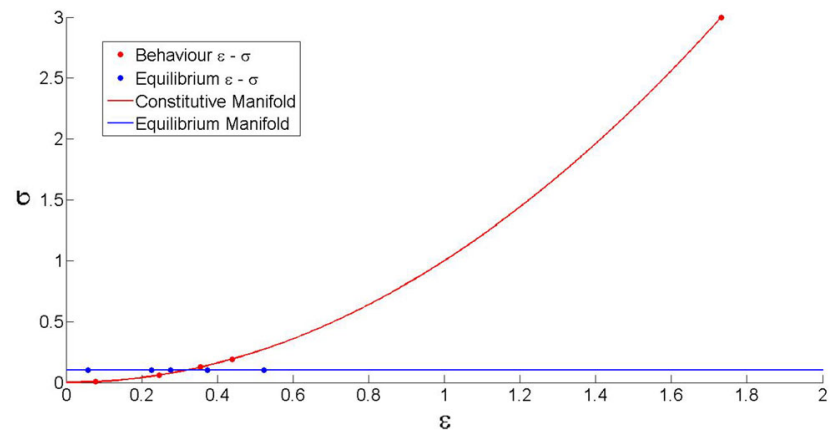

Fig. 5 Beam subjected to a traction for a nonlinear behavior: manifold-based fixed point linearization

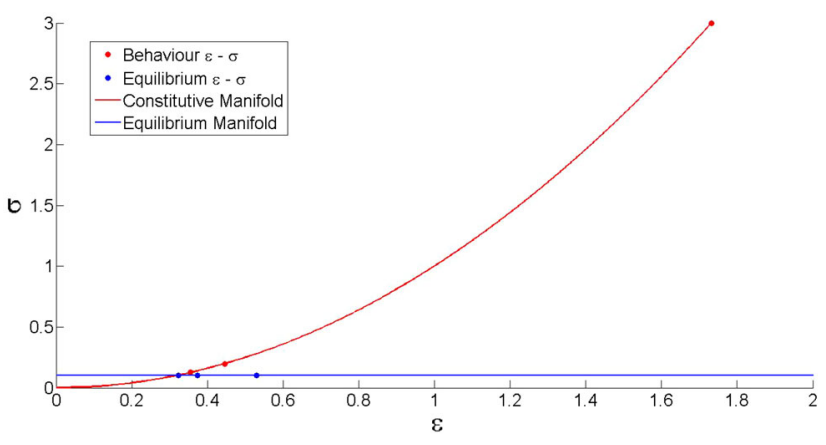

Fig. 6 Beam subjected to a traction for a nonlinear behavior: manifold-based Newton linearization

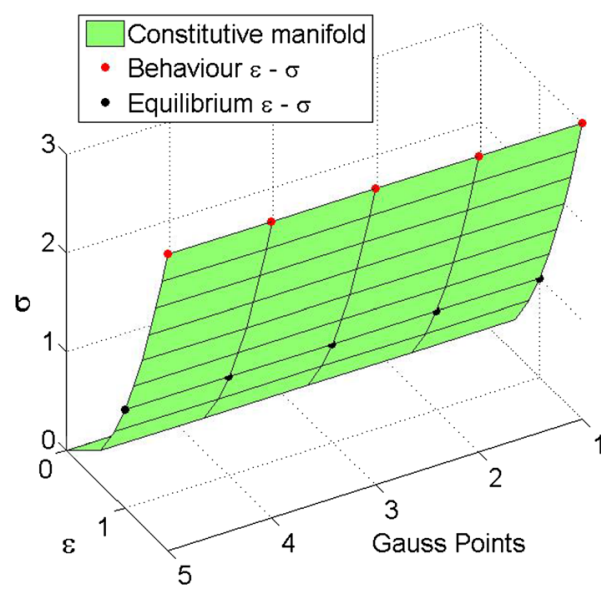

Fig. 7 Beam subjected to traction for a nonlinear behavior

Manifold-based locally linear behaviors resulting in the fixed point and Newton strategies proceed faster that the one based on the solution from the only knowledge of data. However, it requires the identification of such behaviors with the subsequent errors that they could imply if coarse samplings of the constitutive behavior are employed.

\subsection{A Two-Dimensional Case Study}

We considered a 2D problem defined on a square involving again an elastic behavior defined from a manifold in the space $(\boldsymbol{\sigma}, \boldsymbol{\varepsilon})$. This constitutive manifold was proved to project onto a just two-dimensional one in its reduced form, as discussed previously.

The square is clamped on its left boundary, free on the top and bottom sides and a unit traction is applied on its right side. Any of the proposed strategies, the ones making use of the manifold-based locally linear behaviors or the one proceeding directly from data, allow reaching the same converged solution depicted in Fig. 8. The last one employs a single search direction $\boldsymbol{D}$ or a different one at each Gauss point $\boldsymbol{D}(\boldsymbol{x})$. It agrees in minute with the one obtained by 

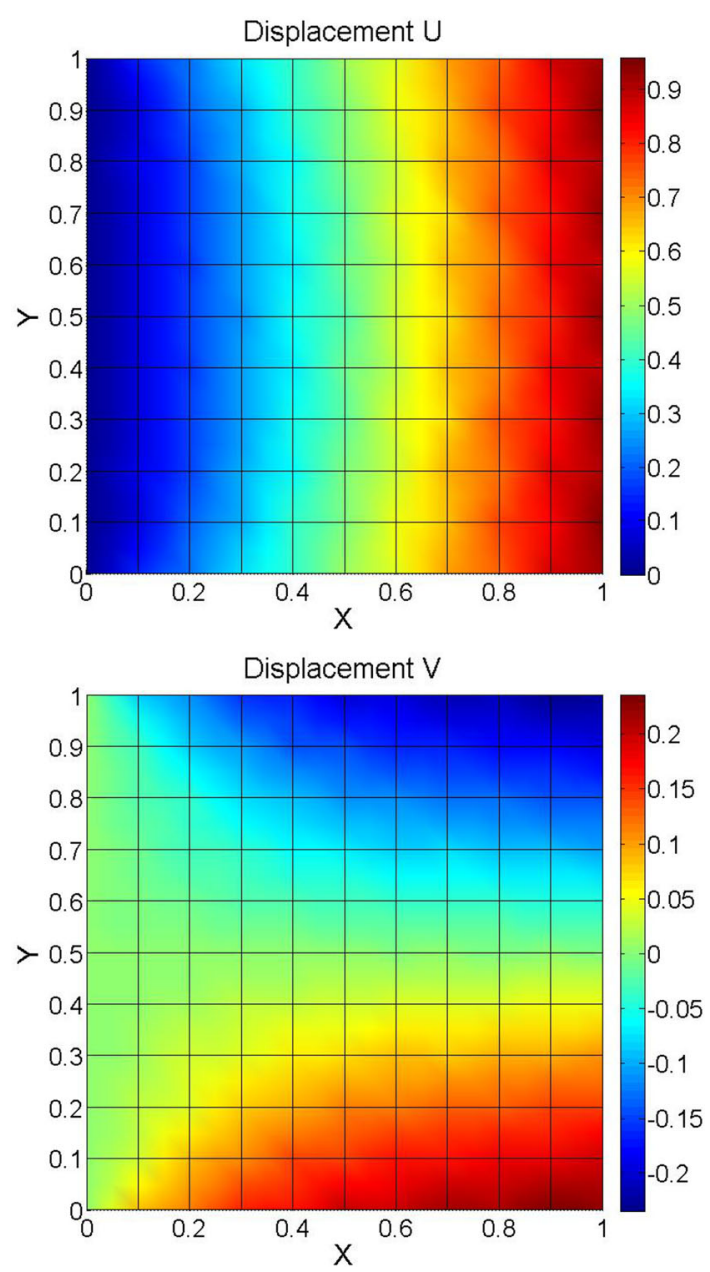

Fig. 8 2D problem associated to a "hidden" linear elastic behavior: (top) horizontal component of the displacement and (bottom) vertical component

using standard model-based discretization. Again, a Newton technique remains superior to the other choices.

In what respects the solution accuracy there are different aspects affecting it: (1) the constitutive manifold sampling when nonlinear behaviors are addressed; (2) the finite element approximation and finally (3) the threshold consider in the nonlinear iteration schemes. Even if a detailed analysis of the accuracy and rate of convergence is beyond the aim of the present work, our numerical experiments indicate that convergence is assured by using fine enough samplings of the constitutive manifolds as well as by considering fine enough finite element discretizations.

\section{Addressing Inelastic Behaviors: Linear Elastic- Perfectly Plastic Behavior}

In this section we start by addressing the case of a linearelastic-perfectly plastic $2 \mathrm{D}$ behavior. We assume the linear elastic contribution defined locally from $\boldsymbol{C}\left(\boldsymbol{X}^{e}\right)$ ( $\boldsymbol{X}^{e}$ refers to the stress-elastic strain manifold) whereas the plastic contribution that involves the yield surface $f(\boldsymbol{\sigma})$ is assumed given by its own manifold.

Using again Voigt notation, the elastic behavior expressed from $\boldsymbol{\sigma}=\boldsymbol{C} \cdot \boldsymbol{\varepsilon}^{e}$, where $\boldsymbol{C}$ represents the manifold-based elastic tensor and $\boldsymbol{\varepsilon}^{e}$ refers to the elastic component of the deformation (the reversible one). The total strain can be decomposed in its elastic and inelastic components,

$\boldsymbol{\varepsilon}=\boldsymbol{\varepsilon}^{e}+\boldsymbol{\varepsilon}^{p}$,

where we assume the plastic flow rate

$\dot{\boldsymbol{\varepsilon}}^{p}=\lambda \frac{\partial f(\boldsymbol{\sigma})}{\partial \boldsymbol{\sigma}}=\lambda \boldsymbol{n}$,

where the yield surface $f(\boldsymbol{\sigma})$ is provided by experimental data. To generate these data in silico, we assume that it follows a von Mises model $f(\boldsymbol{\sigma})=\sigma_{e}-Y$, with $Y$ the yield stress (no hardening is considered) and $\sigma_{e}$ the equivalent stress related to the von Mises criterion. $f(\boldsymbol{\sigma})$ results in the surface represented in Fig. 9 where, for the sake of clarity, it is represented in the space of stresses.

The persistency condition $\dot{f}(\boldsymbol{\sigma})=0$ when plastic flow occurs, results in the following plastic flow

$\lambda=\frac{\boldsymbol{n}^{T} \cdot \boldsymbol{C} \cdot \dot{\boldsymbol{\varepsilon}}}{\boldsymbol{n}^{T} \cdot \boldsymbol{C} \cdot \boldsymbol{n}}$

or in its incremental counterpart

$\lambda=\frac{\boldsymbol{n}^{T} \cdot \boldsymbol{C} \cdot \Delta \boldsymbol{\varepsilon}}{\boldsymbol{n}^{T} \cdot \boldsymbol{C} \cdot \boldsymbol{n}}$,

with now $\Delta \varepsilon^{p}=\lambda \boldsymbol{n}$.

Here three fields must be considered, stress, strain and plastic strain. As soon as the last one is known, the elastic strain can be locally determined and the stresses obtained from the elastic manifold using the couple stress-elastic component of the strain.

In these expressions everything is properly defined except $\boldsymbol{n}$, since we assume that the explicit form of the yield condition, i.e. $f(\boldsymbol{\sigma})$ is unknown and the only available data is the manifold depicted in Fig. 9. However, $\boldsymbol{n}$ is easily

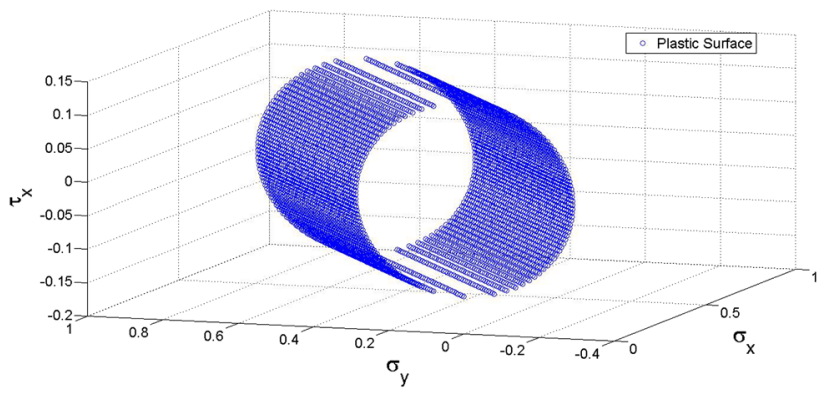

Fig. 9 Plastic manifold associated to the von Mises plasticity case 
accessible by considering the normal vector to the plastic manifold depicted in Fig. 9.

Now one could imagine performing a standard linear elastic-perfectly plastic simulation by using a finite element explicit code where the plastic deformation is computed from the manifold that allows extracting $\boldsymbol{n}$ instead of the knowledge of function $f(\boldsymbol{\sigma})$ and its explicit derivative with respect to the stresses.

When considering the traction of a square domain along its right side, with appropriate boundary conditions on its left side (with tension-free conditions on the top and bottom boundaries) ensuring an homogeneous stress and strain fields everywhere in the domain, the stress trajectory in the

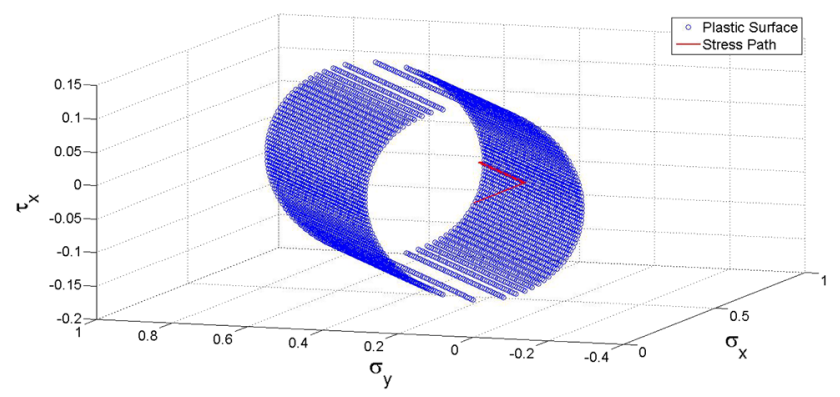

Fig. 10 Stress trajectory in the stress space in the elastic-perfecly plastic behavior stress space is depicted in Fig. 10. It can be noticed that the elastic behavior applies when the stress remains inside the plastic surface and then it remains in the surface during the plastic flow. Again, for the sake of simplicity, the results are shown in the stress domain. Finally, Fig. 11 depicts the three components of the plastic strain for three different levels of the applied load acting on the right side of the clamped square previously considered. The different strategies allows to compute the same results.The Newton algorithm results again to be the one involving less computational effort.

Even if this analysis proved that we could proceed as usually when function $f(\boldsymbol{\sigma})$ is not explicitly known, the elastic behavior was assumed given by the locally-linear elastic manifold. Obviously the extension to implicit formulations or to more complex nonlinear elastic behaviors again based on a locally-linear tangent description is straightforward.

\section{Conclusions}

This paper constitutes a first attempt to reduce the modeling needs in computational mechanics. We proved that by knowing the different stress-strain couples defining the
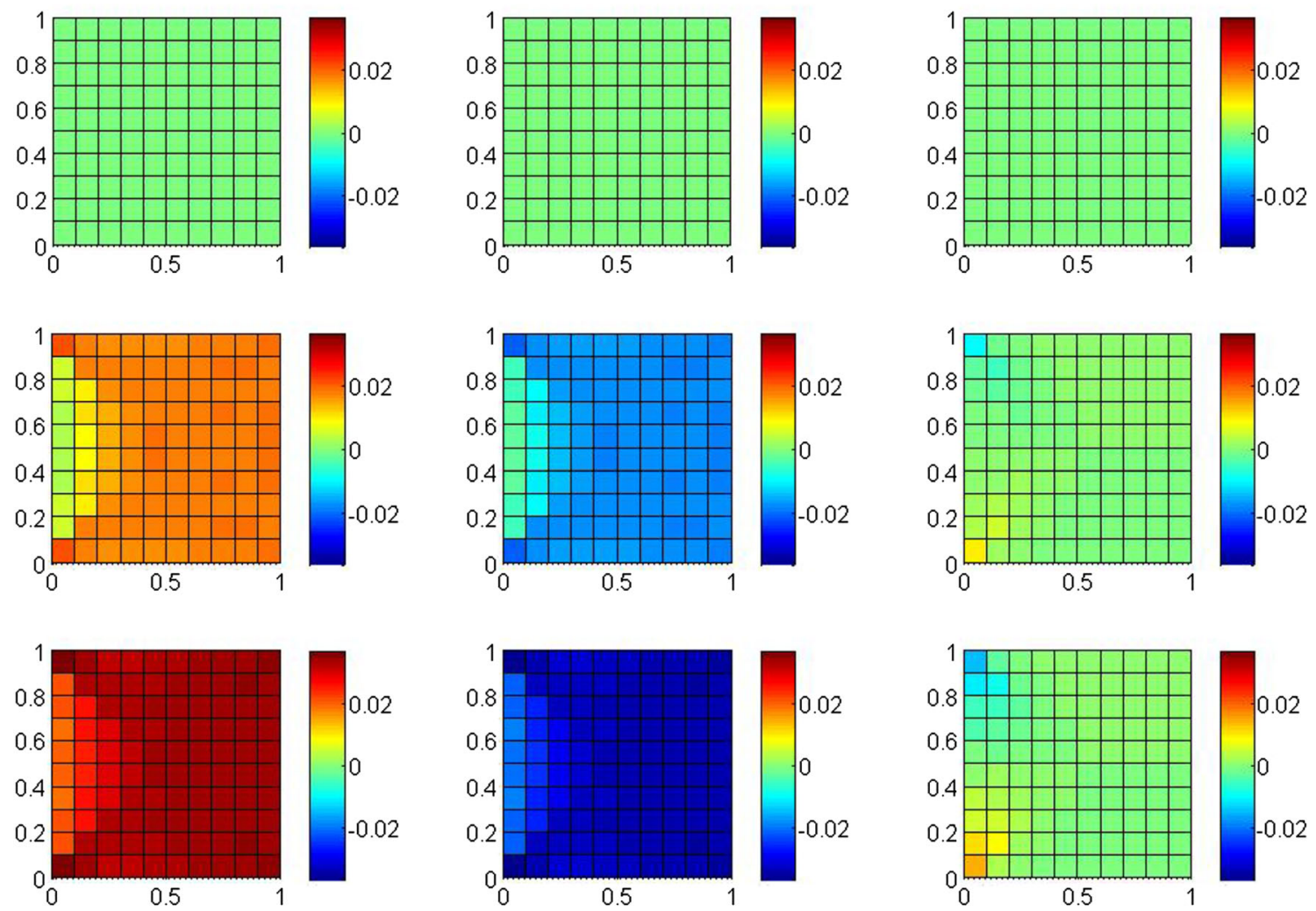

Fig. 11 Plastic strain at the initial time (top), for the half of the total load (middle) and for the entire load (bottom), for components $\epsilon_{x x}^{p}$ (left), $\epsilon_{y y}^{p}$ (center) and $\epsilon_{x y}^{p}$ (right) 
elastic behavior as well as the manifold defining the yield condition there is no need to create models for representing neither the linear or nonlinear elastic behaviors nor the yield condition. Different linearization strategies have been proposed. Two of them are weakly intrusive and easily implantable in existing commercial simulation codes, since they are based on a locally-linear elastic expression. Another linearization strategy proceeding exclusively from data iterates from a local-nonlinear problem to ensure the verification of the constitutive behavior and a linear or nonlinear-global problem for ensuring the mechanical equilibrium.

Despite the fact of addressing quite simple problems, a great potential can be noticed, that could constitute a new paradigm in computational mechanics, linking experimental data with discretization techniques while reducing as much as possible the needs of modeling issues.

Acknowledgments This work has been supported by ESI GROUP through the ECN-ESI Chair on advanced modeling and simulation of materials, structures and processes as well as by the Spanish Ministry of Economy and Competitiveness through Grants Number CICYT DPI2014-51844-C2-1-R and DPI2015-72365-EXP and by the Regional Government of Aragon and the European Social Fund, Research Group T88.

\section{Compliance with Ethical Standards}

Conflict of interest The authors declare that they have no conflict of interest

Human Participants Nor Animals The research does not involve neither human participants nor animals

Informed Consent All the authors are informed and provided their consent.

\section{References}

1. Amsallem D, Farhat C (2008) An interpolation method for adapting reduced-order models and application to aeroelasticity. AIAA J 46:1803-1813

2. Brunton SL, Proctor JL, Kutz JN (2016) Discovering governing equations from data by sparse identification of nonlinear dynamical systems. Proc Natl Acad Sci 113(15):3932-3937
3. Darema F (2005) Grid computing and beyond: the context of dynamic data driven applications systems. Proc IEEE 93(3):692-697

4. González D, Aguado JV, Cueto E, Abisset-Chavanne E, Chinesta F (2016) kpca-based parametric solutions within the PGD framework. Arch Comput Methods Eng. doi:10.1007/s11831016-9173-4

5. González D, Cueto E, Chinesta F (2015) Computational patient avatars for surgery planning. Ann Biomed Eng 44(1):35-45

6. Kirchdoerfer T, Ortiz M (2016) Data-driven computational mechanics. Comput Methods Appl Mech Eng 304:81-101

7. Ladeveze P (1989) The large time increment method for the analyze of structures with nonlinear constitutive relation described by internal variables. Comptes Rendus Académie des Sci Paris 309:1095-1099

8. Lee JA, Verleysen M (2007) Nonlinear dimensionality reduction. Springer, Berlin

9. Liu Z, Bessa MA, Liu WK (2016) Self-consistent clustering analysis: an efficient multi-scale scheme for inelastic heterogeneous materials. Comput Methods Appl Mech Eng 306:319-341

10. Lopez E, Gonzalez D, Aguado JV, Abisset-Chavanne E, Cueto E, Binetruy C, Chinesta F (2016) A manifold learning approach for integrated computational materials engineering. Arch Comput Methods Eng. doi:10.1007/s11831-016-9172-5

11. Michopoulos J, Farhat C, Houstis E (2004) Dynamic-data-driven real-time computational mechanics environment. In: Bubak M, van Albada GD, Sloot PMA, Dongarra J (eds) Computational science-ICCS 2004: 4th international conference, Kraków, Poland, June 6-9, 2004, proceedings, Part III, pp 693-700, Springer, Berlin

12. Olson GB (2000) Designing a new material world. Science 288(5468):993-998

13. Peherstorfer B, Willcox K (2015) Dynamic data-driven reducedorder models. Comput Methods Appl Mech Eng 291:21-41

14. Peherstorfer B, Willcox K (2016) Data-driven operator inference for nonintrusive projection-based model reduction. Comput Methods Appl Mech Eng 306:196-215

15. Polito M, Perona P (2001) Grouping and dimensionality reduction by locally linear embedding. In: Advances in neural information processing systems 14, pp 1255-1262. MIT Press

16. Raghupathi W, Raghupathi V (2014) Big data analytics in healthcare: promise and potential. Health Inf Sci Syst 2(1):1-10

17. Roweis ST, Saul LK (2000) Nonlinear dimensionality reduction by locally linear embedding. Science 290(5500):2323-2326

18. Tenenbaum JB, de Silva V, Langford JC (2000) A global framework for nonlinear dimensionality reduction. Science 290:2319-2323

19. Wang Q (2012) Kernel principal component analysis and its applications in face recognition and active shape models. CoRR, abs/1207.3538 